\title{
Knowledge Base as an Integral Attribute of a Modern Company
}

\author{
Yuriy Golchevskiy*, Andrei Yermolenko
}

\author{
Pitirim Sorokin Syktyvkar State University, Syktyvkar, Russian Federation \\ ${ }^{*}$ Corresponding author. Email: yurygol@mail.ru
}

\begin{abstract}
An integral attribute of a modern company in addition to a corporate website is a knowledge base. Knowledge bases allow significant reduction of operating expenses associated both with core and non-core activities of the company. This study aims at showing the potential for knowledge bases to be used as an IT tool for improvement of company efficiency and competitive advantage. Knowledge bases are considered to be used both for internal and external communication. There is also a focus on the approach to knowledge base design, in particular to data retrieval and customization.
\end{abstract}

Keywords: knowledge base, decision support system, business processes, wiki, corporate site

\section{INTRODUCTION}

Daily activities of an organization are generally connected with routine tasks - employees perform identical operations day after day, they register and submit documents, request information from various departments, create analytical reports, control the production process, etc.

However, conventional work flow may be interrupted by a force majeure, failure of commonly used services, shortage of data or loss of significant information, new employees hired, structural transformations within the organization, etc. The tasks of quick and effective resumption of work, as well as minimizing of these factors are often arise in this regard. In addition, at a certain stage of comprehension of business processes, the problem of using of obtained skills and knowledge, improving teamwork and communicating with customers appears.

One of the solutions for the above and for many other problems may become introduction of knowledge bases into business processes.

A knowledge base is a popular IT tool used by companies for such purposes as:

- Lower workload of support services;

- Incorporation of all corporate information exchange channels into a unified platform;

- Efficient training of new employees;

- Higher efficiency of customer support.

A knowledge base implies more than just a software solution, it is also a technique for storage and processing of useful information, or just a data massif related to a particular field.

Creation of a high-quality knowledge base is quite an expensive process; it requires plenty of time and considerable effort, especially when created manually. In practice, however, it may happen so that the knowledge base brings no benefit. It can be explained by misunderstood meaning and essence of later use of the knowledge base in the organization, wrong choice of technology, etc.

Such errors can be avoided if there is a clear understanding of how knowledge bases are used today, what practical benefits they can bring to a particular organization based on the level of development of its business processes, size, goals and understanding of the potential of this technology in general.

The objective of this work is to present a vision of how knowledge bases can be used as an IT tool to improve organization efficiency and competitive advantage.

\section{RESEARCH METHODS}

This study is based on the analysis of modern trends, the analysis of publications and scientific discussions on this topic. We also used the methods of observation and comparison based on data obtained from many years of work experience, methods of interviewing representatives of employers and professional communities, university graduates.

\section{RESULTS}

\subsection{Practical application areas of knowledge bases for business improvement}

Let us describe how knowledge bases can be used in a company. The most obvious, fairly simple and welldeveloped solution is to use knowledge bases to reduce the 
work load of the support and training service. According to the research done by Destination CRM (https://www.destinationcrm.com/), about $2 / 3$ of customers ask the same questions related to operation of a particular service or product. Availability of a high-quality knowledge base with a well-thought-out interface at a company's website can allow users to find answers independently or reduce the staff load when answering and finding solutions to user problems.

The possibility to train entrepreneurs through knowledge management technologies based on a mobile application for small and medium-sized businesses is reviewed in [1], while approaches and practices for personnel training are presented, for instance, in such papers as [2-4], which highlights the variety of methods for the technology application.

In order to be competitive, companies and organizations have to analyze and improve their business processes continuously. For example, the article [5] presents a number of attributes and cases leading to revision of business processes. It is an extraordinary task requiring knowledge and extensive experience in business process reengineering. In such cases, computer-based design and knowledge base operation tools can be used.

The article [6] describes a method for knowledge base organization based on the use of business process templates. It shows a repository consisting of business process templates that are thoroughly tested at the syntactic and semantic levels and a template management mechanism, and proposes a process for workflow template development. Thus, users can select and modify corresponding workflow templates from the knowledge base. The paper [7] presents a platform for business processes knowledge base management, which incorporates a logical language for business process modeling and a reasoning mechanism that provides support to designers.

Feasibility and effectiveness of the method of presenting and building knowledge about product requirements were justified in [8] on the example of the knowledge base system for a mobile website.

Another area where companies can apply ideas of knowledge base design and use effectively is generation of various business analytics $[9,10]$. Retrieval and analysis of a wide range of business information from various sources, including scientific ones, various web resources, and social networks, creation of a company's business profile - these tasks can be performed by data processing systems with knowledge bases connected to them.

Knowledge base application directly at a production area can also be relevant. It is a useful tool for optimizing product or service production or design processes through increased productivity and efficiency of reused information and knowledge in the course of simultaneous data analysis and machine learning algorithms.

An example of a possible solution for the polyethylene production process is given in the article [11], and a review of technologies for the intelligent design of equipment and a knowledge base management system, which can save a lot of time to designers by my means of reused design knowledge, is given in [12].
Software development is no exception as well. A study on development of a support system for a software design phase based on the findings of security requirements and knowledge base analysis is presented in [13].

Software solutions based on a dynamic and expandable knowledge base can determine optimal implementation of working processes with existing equipment configurations, performing the role of data storage and functions of a human analytical expert. A draft solution to support compilation of high-level languages for arbitrary reconfigurable platforms was presented in [14].

In addition, knowledge bases serve as the foundation for development of technologies of cognitive robots and machine learning systems.

And, of course, one cannot but mention the use of knowledge bases in decision support systems at various levels ranging from systems aimed at improving the efficiency of small businesses, social and healthcare organizations to industry-related systems and municipal, regional and state level decision support systems [15 - 21].

\subsection{Practical aspects to consider}

Having analyzed the needs and potential of a knowledge base use in an organization, it is necessary to consider feasibility of its development and select an implementation method. There is a wide range of options for technical implementation of such a knowledge base - from a simple collection of documents to complex hierarchical structures supported by a variety of data acquisition and processing algorithms, including big data and machine learning processing, speech recognition and ontology creation. The resulting product can be fully automated, supported by a company's structural unit or dedicated employees of various departments.

When designing a system, several important questions have to be answered. First of all, it is necessary to determine what kind of information will be accumulated and processed in the database (product data, business analytics, production parameters, etc), who will use it (employees or customers, for example), and whether it should be further structured for report generation, etc.

It is important to understand that creation of a knowledge base and initiated information entry into it are just a number of obvious aspects of the immense work. Since there has been tremendous growth in the amount of information in recent years, it causes a risk that the database will grow quickly and the search for relevant information may become problematic, as can be observed with regard to knowledge bases in some large IT corporations. Therefore, even at the design stage, a primary focus should be given to how content classification and search will be implemented in it. Otherwise the project may simply turn out to be useless. The third important aspect that requires a well-thought decision is whether to create a system on your own or based on existing solutions. The answer, indeed, is not easy, since many of the existing solutions do not offer sufficient customization, which immediately leads to their futility and causes a lot of problems with data compilation and analysis. 
Medium and large companies can afford their own development, and sometimes it becomes merely essential for any further development. For small businesses, it will be more practical to use off-the-shelf solutions making it possible to create a single workflow, at least at initial stages.

\subsection{Knowledge base implementation options for small business}

Corporate and Wiki websites are popular and easy-to-use means of knowledge base implementation. See some examples of such solutions below:

1. Confluence (https://www.atlassian.com)

The developer is Atlassian. Confluence is a teamwork space based on a replicated Wiki-system designed for internal use by organizations in order to create an integrated corporate space and knowledge base. It is, in general, a fairly simple and user-friendly tool, but, in our opinion, it has insufficient customization and problems with navigation and search.

2. Notion (https://www.notion.so/)

The developer is Notion Labs Inc. This product combines a sufficiently large number of tools - notes, to-do lists, documents, kanban boards and knowledge bases, everything an office employee usually has to deal with into a single workspace. You can add various elements to it and assemble an "ideal" tool for data storage, planning and teamwork. However, it is a cloud service with all respective disadvantages.

3. Microsoft Sharepoint platform. Its distinctive features include the ability to use a wide variety of tools and compatibility with other Microsoft products. The platform has extensive customization options for the needs of a company, ensuring stable operation and performance. In 2014, the Sochi Winter Olympics steering committee introduced a base on this platform. The structure was based on a simple scheme, which included functionalities characterizing briefly the essence of the data: "Nutrition", "Communication with the Press", "Health Care", etc. The shortcomings of this technology include complicated implementation of a corporate database at Sharepoint, insufficient integration with third-party software, and the cost of the solution. As a consequence, such a solution is not very suitable for small businesses.

A similar benchmarking of over 20 most popular Wiki systems can be performed with the help of [22].

Also, tools based on the use of applications for mobile devices and SaaS services have recently emerged and are being developed. Among the proposals are models and software solutions for knowledge representation and modification in the form of logical rules and their further interpretation [23], a multidimensional model for optimized filling of the data store based on reporting requirements [24], a unified approach to managing knowledge bases of various configurations and development of unified mathematical models of operations with ontology elements [25].

\section{CONCLUSION}

Under current conditions of information prevalence, we are observing increased relevancy of more efficient corporate and public administration through full-featured application of technological and information capacities ensuring data accumulation, analysis, assessment and reuse, with subsequent forecasting of changes in the situation and information and analytical support for the decision-making process. "Information overload" with its complexity of management processes and a significantly increased volume of analyzed data caused by their redundancy, irrelevance, selectivity, tendentiousness, etc., can lead to incorrect management decisions. One of the solutions is development and implementation of knowledge bases and later - decision-making support systems based on the knowledge presented. The field of application of such tools is extensive - from small businesses, to industries and public administration, from providing an easy-to use common corporate space to creating analytics and generating recommendations. To summarize, we would like to focus on the following:

1. When designing and developing knowledge bases, it is important to answer three relevant questions about the purpose, structure and development/purchase of such a software product correctly.

2. Someone should be responsible for the knowledge base. It cannot run "by itself". The knowledge base requires financial and other costs to be maintained and developed.

3 . For minor projects, you can get ready-made out-of-thebox or cloud solutions, but you have to understand that with large amounts of information such solutions will not operate properly.

4. One of the most important functionalities in the knowledge base is the ability to search. In the long run, the efficiency of the system will be determined by the quality of the search service developed.

\section{REFERENCES}

[1] J.J. Siregar, R.A. Aryanti Wardaya Puspokusumo. Design and development of knowledge management system in the small and medium-scale enterprises base on mobile apps (SMEs at indonesia) (2020) Advances in Intelligent Systems and Computing, 1073, pp. 1020 1030. DOI: https://doi.org/10.1007/978-3-030-335823_96

[2] Eu. Khalin, Ye. Mikhaylova. Application of intelligent graphics for ensuring electrical safety of production. Research in Agricultural Electric Engineering, 2016, no 1, pp. 8- 14

[3] G.S. Sigovtsev, I.O. Semenov, M.A. Charuta. LCMS for personnel training based on the corporate knowledge base. Modern information technologies and 
management system for intelligent outfitting design (2019) ACM International Conference Proceeding Series, pp. 62 - 66. DOI: https://doi.org/10.1145/3341069.3341078

organization management tool. Economic development
o.V. Yermolenko. Knowledge base as an in the context of digital transformation. Collection of scientific papers of the All-Russian national scientific and practical conference. Syktyvkar, Syktyvkar State Univ., 2018, pp. 25 - 29 (In Russ.)

[5] N. Kettler, P. Soffer, I. Hadar. Towards a Knowledge Base of Business Process Redesign: Forming the Structure (2019) Lecture Notes in Business Information Processing, 352, pp. 3 - 18. DOI: https://doi.org/10.1007/978-3-030-20618-5_1

[6] T.H.H. Nguyen, T.P. Hong, N. Le Thanh. An ontological approach for organizing a knowledge base to share and reuse business workflow templates (2017) 7th International Conference on Information Science and Technology, ICIST 2017 - Proceedings, pp. 271 277. DOI: https://doi.org/10.1109/ICIST.2017.7926769

[7] A. De Nicola, M. Missikoff, M. Proietti, F. Smith. A logic-based method for business process knowledge base management (2010) SEBD 2010 - Proceedings of the 18th Italian Symposium on Advanced Database Systems, pp. $170-181$

[8] B. Lei. Construction of product requirements knowledge base on E-business website (2015) Jisuanji Jicheng Zhizao Xitong/Computer Integrated Manufacturing Systems, CIMS, 21 (3), pp. 618 - 625. DOI: https://doi.org/10.13196/j.cims.2015.03.006

[9] S. Shin, H. Jung, M.Y. Yi. Building a business knowledge base by a supervised learning and rule-based method (2015) KSII Transactions on Internet and Information Systems, 9 (1), pp. 407 - 420. DOI: https://doi.org/10.3837/tiis.2015.01.025

[10] D. Consoli, D. Elche. An analysis of the knowledge base of scientific research and development business services (2014) R and D Management, 44 (4), pp. 341 - 354. DOI: https://doi.org/10.1111/radm.12062

[11] W. Zhong, C. Li, X. Peng, F. Wan, X. An, Z. Tian, A Knowledge Base System for Operation Optimization: Design and Implementation Practice for the Polyethylene Process (2019) Engineering, 5 (6), pp. 1041 - 1048. DOI: https://doi.org/10.1016/j.eng.2019.09.004

[12] Y. Ji, X. Hu, J. Liu, Z. Yang, L. Wang, Z. Jiang.Design and application of knowledge base
[13] A. Hazeyama, H. Miyahara, T. Tanaka, H.Washizaki, H. Kaiya, T. Okubo, N. Yoshioka. A system for seamless support from security requirements analysis to security design using a software security knowledge base (2019) Proceedings - 2019 IEEE 27th International Requirements Engineering Conference Workshops, REW 2019, pp. 134 - 140. DOI: https://doi.org/10.1109/REW.2019.00029

[14] H. Zhou, A. Srivastava, R. Kannan, V. Prasanna. Design and implementation of knowledge base for runtime management of software defined hardware (2019) IEEE High Performance Extreme Computing Conference, HPEC 2019. DOI: https://doi.org/10.1109/HPEC.2019.8916328

[15] D.L. Olson, V. Van Huy, N.M. Tuan. Case of development of a small business ERP consultant knowledge base (2012) Advances in Enterprise Information Systems II - Proceedings of the 5th International Conference on Research and Practical Issues of Enterprise Information Systems, CONFENIS 2011, pp. $81-90$

[16] W.A. Teniwut, C.L. Hasyim. Decision support system in supply chain: A systematic literature review (2020) Uncertain Supply Chain Management, 8 (1), pp. 131 - 148. DOI:

https://doi.org/10.5267/j.uscm.2019.7.009

[17] M. Zhang, G. Tian, J. Gong, Y. Yuan. Design method of service decision mechanism based on ontology knowledge base (2017) Huazhong Keji Daxue Xuebao (Ziran Kexue Ban)/Journal of Huazhong University of Science and Technology (Natural Science Edition), 45 (10), pp. 70 - 74. DOI: https://doi.org/10.13245/j.hust.171013

[18] C. Zhang, F. Sun, M. Zhang, W. Liu, Q. Yu, P. Babyn, H. Zhong. Design and implementation of a medical image knowledge base for pulmonary nodules diagnosis (2018) 2017 3rd IEEE International Conference on Computer and Communications, ICCC 2017, 2018 - January, pp. 2071 - 2075. DOI: https://doi.org/10.1109/CompComm.2017.8322901

[19] Z. Zhai, J.F. Martínez, V. Beltran, N.L. Martínez. Decision support systems for agriculture 4.0: Survey and challenges (2020) Computers and Electronics in 
[23] A.Y. Yurin, N.O. Dorodnykh. Personal knowledge base designer: Software for expert systems prototyping (2020) SoftwareX, vol. 11, DOI:

https://doi.org/10.1016/j.softx.2020.100411

Conceptual framework of an intelligent decision support system for smart city disaster management (2020) Applied Sciences (Switzerland), 10 (2). DOI: https://doi.org/10.3390/app10020666

[21] A.Yu. Bersenev, Yu.V. Golchevskiy. Situational centers using in solving actual public administration problems. Information technologies in modeling and management: approaches, methods, solutions,

Collection of scientific papers of the 2-nd All-Russian scientific conference with international participation. Part 2, Togliatti, Togliatti State Univ., 2019, pp. 329 337 (In Russ.)

[22] Compare Them All | WikiMatrix. Available at: https://www.wikimatrix.org/
[24] A. Azzini, S. Marrara, A. Maurino, A. Topalović. A report-driven approach to design multidimensional models (2019) Lecture Notes in Business Information Processing, 340, pp. $105-127$. DOI: https://doi.org/10.1007/978-3-030-11638-5_6

[25] I. Kotov, O. Suvorov, O. Serdiuk. Development of methods for structural and logical model unification of metaknowledge for ontologies evolution managing of intelligent systems (2019) Eastern-European Journal of Enterprise Technologies, 2 (4 - 98), pp. 38 - 47. DOI: https://doi.org/10.15587/1729-4061.2019.155410 